\title{
The problem 4 of placement triangular geometric line field
}

\author{
Vladimir Iljich Travush ${ }^{1}$, Vasilij Dmitrievich Antoshkin ${ }^{2, *}$, Vladimir Trofimovich \\ Erofeev $^{2}$ \\ ${ }^{1}$ RAASN, Bolshaya Dmitrovka 24-1,Moscow,107031, Russia \\ ${ }^{2}$ Mordovian State University, Bolshevistskaya 68, Saransk,430005, Russia
}

\begin{abstract}
One of the a method of formation of triangular networks in the field is investigated. Conditions the problem of locating a triangular network in the area are delivered. The criterion for assessing the effectiveness of the solution of the problem is the minimum number of sizes of the dome elements, the possibility of pre-assembly and prestressing. The solution of the problem of one embodiment of a triangular network of accommodation in a compatible spherical triangle and, accordingly, on the sphere. Optimization of triangular geometric network on a sphere on the criterion of minimum sizes of elements can be solved by placing the system in an irregular hexagon inscribed in a circle of minimal size, maximum regular hexagons.
\end{abstract}

\section{Introduction}

Placing on the area of regular and irregular hexagons, inscribed in a circle, ie, flat figures or composed in turn of spherical triangles (see Fig. 1) with minimum dimensions of the ribs, is an effective solution in the form of a network formed by circles of minimum radii, ie, circles on a sphere obtained at the touch of three adjacent circles whose centers are at the shortest distance from each other [1-3. 5-8]. Optimization of triangular geometric network on a sphere by the criterion of minimum sizes of elements may be presented and solved by placing the system of irregular hexagon, inscribed in the minimum size of the circle, the maximum regular hexagons, for example, compatible spherical triangles (segments) icosahedron with the scheme of cutting, as shown in Figures 1 and 2. In the application options of the symmetry properties of the main and parallel lines of circles spheres, in such cutting must be realized the possibility of central symmetry of circles [1-4].

\section{The problem 4 of placement triangular geometric line field.}

The diagrams Figures 1 and 2 shows the placement of the circumscribed circle of the hexagons in a spherical triangle (compatible spherical segment of the icosahedron) with

\footnotetext{
* Corresponding author: antovd@mail.ru
} 
internal angles of 36,90 and $60^{\circ}$. When optimizing the network using a triangular symmetry of circles and spheres of major lines can be identified as one of the intermediate objectives - determining the position of the first rows of hexagons centers $\mathrm{O}_{1}, \mathrm{O}_{2}, \mathrm{O}_{3}, \mathrm{O}_{4}$ (Fig. 2). Accommodation centers of circles describing irregular and regular hexagons, are conducted in the cutting of a 3920-polyhedron (Fig. 1). So, at the first stage we determine the position $\mathrm{O}_{1}$ centers, $\mathrm{O}_{2}, \mathrm{O}_{3}, \mathrm{O}_{4}$ hexagons equal to the radius in the spherical triangle (Fig. 2 shows part of the triangle-segment have interior angles of 60 and 90 degrees). There is a rectangular spherical triangle with internal angles of 90 and 60 degrees and the leg equal to a. Required to determine the values of the radii between the centers $\mathrm{O}_{1}$ and $\mathrm{O}_{2}, \mathrm{O}_{3}$ and $\mathrm{O}_{1}, \mathrm{O}_{1}$ and $\mathrm{O}_{4}$.

To solve this problem, we consider a half regular hexagon $\mathrm{O}_{1}, \mathrm{O}_{2}, \mathrm{O}_{3}, \mathrm{O}_{4}$ and the rightangled triangle $\mathrm{O}_{2} \mathrm{O}_{1} M$ with the leg $\mathrm{r}_{0}$ and the hypotenuse is equal to the total radius $\mathrm{r}_{1}+\mathrm{r}_{2}$ and right triangle $\mathrm{O}_{2} \mathrm{O}_{0} M$, legs $r_{0}$ and $x$, and

$$
x=a-2 r_{0}
$$

Based on the fundamental formulas of spherical trigonometry, we get the system of equations (1 and 2).

Applying the theorem of cosines and sines to the triangle $\mathrm{O}_{2} \mathrm{O}_{0} \mathrm{M}$ we get

$$
\begin{gathered}
\operatorname{tg} y=\frac{\operatorname{tg}\left(a-2 r_{0}\right)}{\cos 60^{\circ}}, \\
\cos y=\cos \left(a-2 r_{0}\right) \cos r_{2}, \\
\sin y=\frac{\sin r_{2}}{\sin 60^{\circ}} .
\end{gathered}
$$

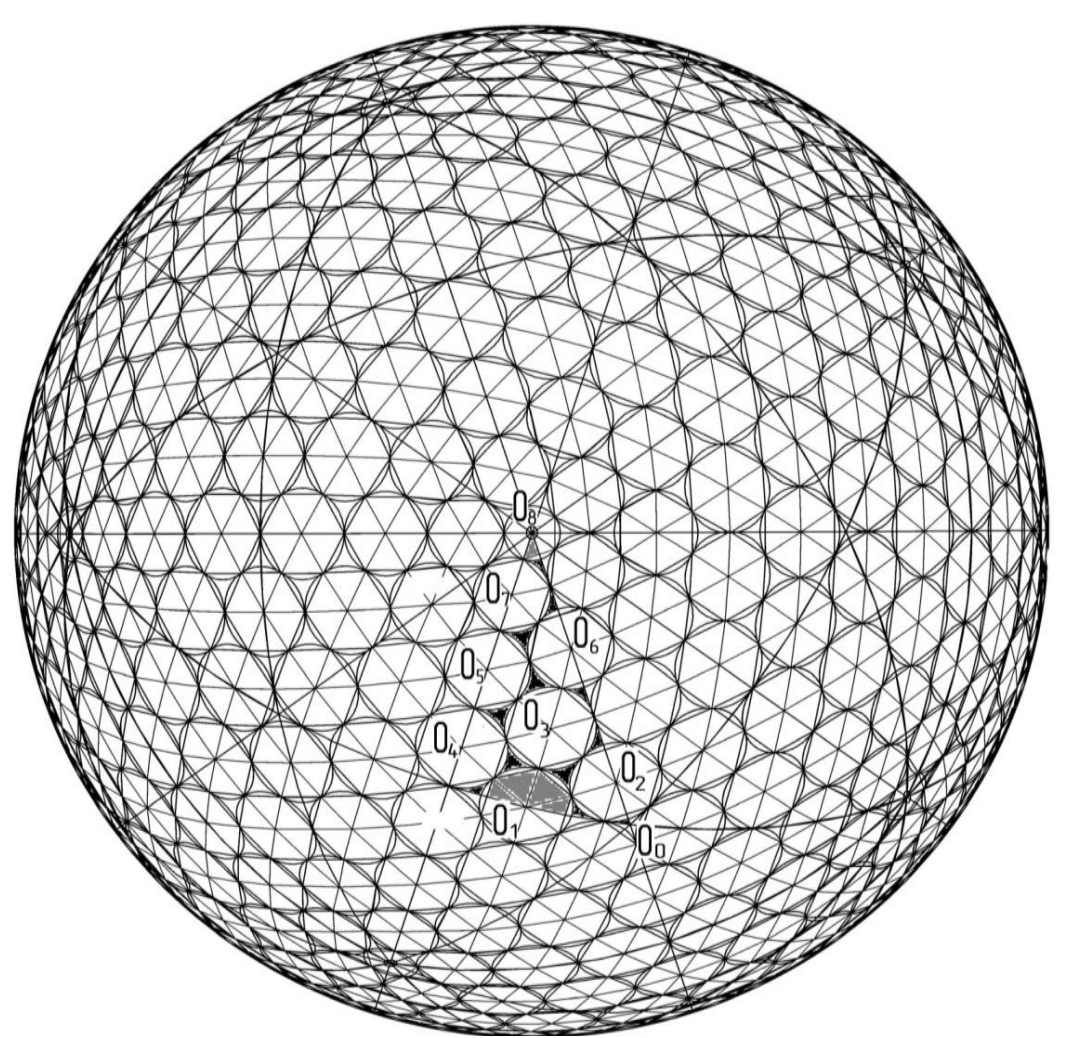

Fig. 1. A sphere made up of compatible spherical triangles 
A sphere made up of compatible spherical triangles (segments, representing 1/6 of the brink of the icosahedron), by cutting a triangular network of 3920-polyhedron having sizes of 20 elements or mounting elements 7 sizes (all sizes -15 ); $\mathrm{O}_{0}$ - center of the face of the icosahedron and the panel in the form of an equilateral triangle on the sphere; $\mathrm{O}_{1}, \mathrm{O}_{2}, \mathrm{O}_{3}, \mathrm{O}_{4}$ be the centers of the mounting panels in the form of flat hexagons and pentagons in a segment of the internal angles of $36,90,60^{\circ}$ for solving the optimization problem of the triangular network

Whence

$$
\frac{\operatorname{tg}\left(a-2 r_{0}\right)}{\cos 60^{\circ}}=\frac{\sin r_{2}}{\sin 60^{\circ} \cos \left(a-2 r_{0}\right) \cos r_{2}} .
$$

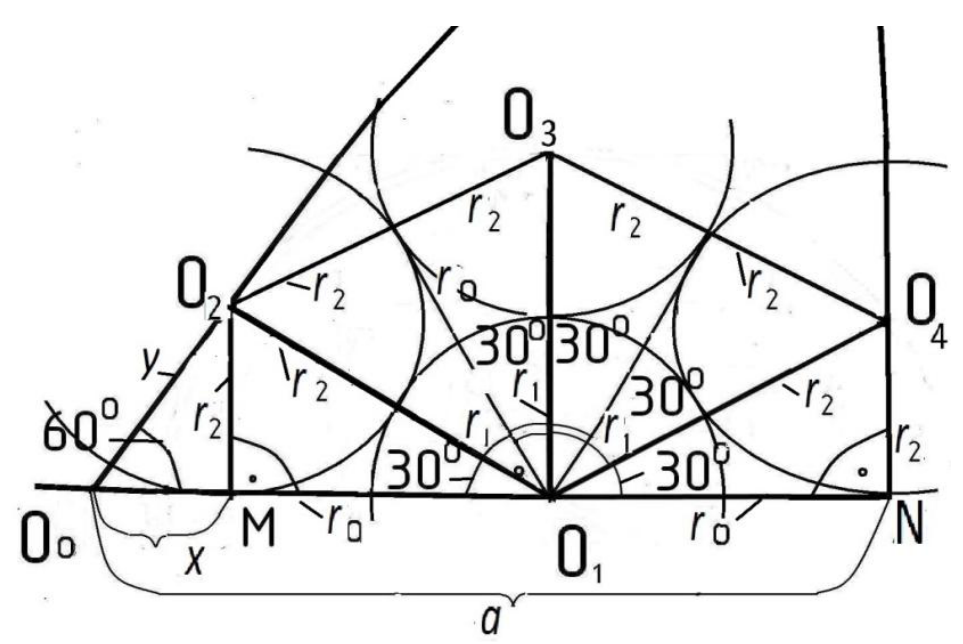

Fig. 2. Scheme of placement

Scheme of placement of the first four flat hexagonal in segment with the inner corners 36 , $90,60^{\circ}$ for solving the optimization problem of the triangular network using the symmetry properties of circles and spheres of major lines (main lines are axes within a spherical segment): $\mathrm{O}_{1}, \mathrm{O}_{2}, \mathrm{O}_{3}, \mathrm{O}_{4}$ - the centers of the panels in the form of flat hexagons

Applying the theorem of cosines and sines to the triangle $\mathrm{O}_{2} \mathrm{O}_{1} \mathrm{M}$ we get

whence

$$
\begin{gathered}
\operatorname{tg}\left(r_{1}+r_{2}\right)=\frac{\operatorname{tg} r_{0}}{\cos 30^{\circ}} \\
\cos \left(r_{1}+r_{2}\right)=\cos r_{0} \cos r_{2}, \\
\sin \left(r_{1}+r_{2}\right)=\frac{\sin r_{2}}{\sin 30^{\circ}}
\end{gathered}
$$

$$
\frac{\operatorname{tg} r_{0}}{\cos 30^{\circ}}=\frac{\sin r_{2}}{\sin 30^{\circ} \cos r_{0} \cos r_{2}} \text {. }
$$

Through transformation of the equations $(1,2)$, we obtain the equation

$$
\operatorname{tg} r_{2}=\cos 30^{\circ} \sin 60^{\circ} \sin \left(a-2 r_{0}\right)=\cos 60^{\circ} \sin 30^{\circ} \sin r_{0}
$$

From equations (3) we obtain

$$
3 \sin \left(a-2 r_{0}\right)=\sin r_{0}
$$


through transformation of the equation (4), we obtain the equation

$$
\begin{gathered}
3\left(\sin a \cos 2 r_{0}-\cos a \sin 2 r_{0}\right)=\sin r_{0} \\
\left.\left(3 \sin a-\sin r_{0}\right)-6 \sin a \sin ^{2} r_{0}\right)^{2}=36 \cos ^{2} a\left(1-\sin ^{2} r_{0}\right) \sin ^{2} r_{0}, \\
9 \sin ^{2} a-6 \sin a \sin r_{0}+\sin ^{2} r_{0}-12 \sin a \sin ^{2} r_{0}\left(3 \sin a-\sin r_{0}\right)+36 \sin ^{2} a \sin ^{4} r_{0}= \\
=36 \cos ^{2} a \sin ^{2} r_{0}-36 \cos ^{2} a \sin ^{4} r_{0}
\end{gathered}
$$

So we get the equation

$$
\begin{gathered}
9 \sin ^{2} a-6 \sin a \sin r_{0}+\sin ^{2} r_{0}-36 \sin ^{2} a \sin ^{2} r_{0}+12 \sin a \sin ^{3} r_{0}++36 \sin ^{2} a \sin ^{4} r_{0} \\
\quad=36 \cos ^{2} a \sin ^{2} r_{0}-36 \cos ^{2} a \sin ^{4} r_{0} \\
9 \sin ^{2} a-6 \sin a \sin r_{0}+\left(1-36 \sin ^{2} a-36 \cos ^{2} a\right) \sin ^{2} r_{0}+12 \sin a \sin ^{3} r_{0} \\
\quad+36 \sin ^{2} a \sin ^{4} r_{0}+36 \cos ^{2} a \sin ^{4} r_{0}=0 \\
9 \sin ^{2} a-6 \sin a \sin r_{0}-35 \sin r_{0}{ }^{2}+12 \sin a \sin ^{3} r_{0}+36 \sin ^{4} r_{0}=0 \\
36 \sin ^{4} r_{0}+12 \sin a \sin r_{0}{ }^{3}-35 \sin ^{2} r_{0}-6 \sin a \sin r_{0}+9 \sin ^{2} a=0
\end{gathered}
$$

Introducing auxiliary notation

$$
k=\sin a, \quad t=\sin r_{0},
$$

and getting rid of irrationality, we turn up to the equation

$$
36 t^{4}+12 k t^{3}-35 t^{2}-6 k t+9 k^{2}=0 .
$$

Spending estimates for the value of leg $a=20.9051628^{\circ}$, we get

$$
\begin{gathered}
36 t^{4}+4,281866124 t^{3}-35 t^{2}-2,14093306 t+1,145898594=0 . \\
\sin r_{0}=0,15581464 . \\
r_{0}=8,9640458 .
\end{gathered}
$$

Then the relations connecting radii $r_{1}$ and $r_{2}$ with parameter $r_{0}$ :

$$
\begin{gathered}
\operatorname{tg} r_{2}=\cos 60 \sin ^{\circ} 30^{\circ} \sin r_{0}, \\
\sin \left(r_{1}+r_{2}\right)=\frac{\sin r_{2}}{\sin 30^{\circ}}
\end{gathered}
$$

We obtain the required values $r_{1}=5.1824133^{\circ}$ and $r_{2}=5.1404432$.

To solve the equation (6) we ve used free software Sclab 5.4.1 - The free platform for Numerical Computation (analogue Matlab) [9].

For the got position of centers of the first rows of the hexagons entered in circumferences, for them corners, sizes of parties of hexagons, are already certain. Accepting the location of cathetus with the size of arc, equal a, on the equator of sphere, we use for the construction of circumferences for other hexagons symmetry of main circumferences (lines) spheres that with a step, equal $\mathrm{r} 0$, we range athwart to this equator [10-12]. When approaching the Pentagon internal symmetry is broken and the circumference is slightly shifted relative to these axes. Calculating the position of the centres of circles in these cases, produced by the method of triangulation. The changes of corners of internal corners of ribs-parties, and and optimization, are possible here for hexagons with centers $\mathrm{O}_{5}-\mathrm{O}_{7}[10-12]$. 


\section{Conclusions}

These solutions allow to implement the scope of approximation algorithms triangular geometric network with the maximum number of regular hexagons and prepare options for optimization of cutting sphere.

\section{References}

1. V.D. Antoshkin, V.T. Erofeev, V.I. Travush, V.I. Rimshin, V.L. Kurbatov, Modern Applied Science. 9(3), 46-50 (2015)

2. V.D. Antoshkin, Regional architecture and engineering. 3 (24), 112-121 (2015)

3. C.A. Bretschneider. Arch. Math. 2. 225-261 (1842)

4. G. A. Korn Mathematical handbook: For scientists and engineers, (1968)

5. W. Lienhard, Elem. math. 66(2) 74-82 (2011)

6. W. J. M'Clelland, T. Preston. A treatise on spherical trigonometry with application to spherical geometry and numerous examples. P. II. (London: Macmillian and Co. 1886)

7. B.V. Miryaev B.V. Regional architecture and engineering, 3, 122-125 (2012)

8. V. F. Manukhov, A. S. Turchin, Glossary of geodetic terms: proc. manual (Publishing Mord. Univ. Saransk, 2005)

9. Software Scilab 5.4.1 - The free platform for Numerical Computation/ Date Views 06.17.20

10. V. I. Travush, V.D. Antoshkin, I.V. Yerofeyeva, D.V. Antoshkin. TEAM spherical shell Patent RU № 2564545 07/28/2014 year

11. V. I. Travush, V.D. Antoshkin, V.T. Erofeev, S.S. Gudozhnikov. Construction and reconstruction. 2012. № 6 (44). pp. 45-55. 